\title{
UNA MIRADA DE LA INFANCIA DESDE EL ENTE RECTOR DEL FÚTBOL INFANTIL EN EL URUGUAY
}

Uma mirada da infância desde o ente reitor do futebol infantil no Uruguai

A glance at the childhood from the governing body of kid's football in Uruguay

\section{Liber Nicolás Benítez González}

Universidad de la República. Instituto Superior de Educación Física. Departamento Académico Educación Física y Deporte. Contacto: 098645607 / liberbenitez.86@gmail.com

\section{Resumen}

En el marco de la relación que históricamente ha mantenido la educación física con el deporte, se hace difícil no atender el significado del fútbol infantil como práctica cultural y fenómeno social en relación con la política pública en la agenda de investigación actual. El deporte aparece como práctica cultural, a la vez que se presenta fundamento recurrente al momento de hablar de desarrollo social y de políticas públicas. En este sentido se torna relevante visualizar los diversos intereses que lo atraviesan, desde las facetas política, económica, educativa hasta el gran nivel de convocatoria e impacto que supone la práctica deportiva en sus diferentes formas de expresión -como espectáculo, como práctica profesional o de deporte en el tiempo libre en una primera división dinámica e interactuante-. Siguiendo las ideas propuestas por Dunning (Elías y Dunning, 1986), la imagen del deporte es idealizada y mistificada separándose del terreno social que le otorga sentido, por lo que en este caso se propone realizar un análisis del deporte en general, y del fútbol infantil en particular como agencia social. El presente trabajo se enmarca en el proyecto de investigación "Fútbol infantil y gobierno de la infancia. La Organización Nacional del Fútbol Infantil en el Uruguay (1968-2015)”, que propone generar un estudio descriptivo en relación al fútbol infantil en tanto gobierno de la infancia, que parta del análisis de las nociones de cuerpo y deporte para poder identificar qué deporte para qué infancia en el fútbol infantil.

Palabras clave: fútbol infantil, infancia, gobierno, deporte. 


\begin{abstract}
In consideration of the relationship that historically has maintained the physical education with sports, it's hard not to address the meaning of kid's football as a cultural and social phenomena in relation with public policies. The sport is a cultural practice, which in many cases is used as a fundament to establish policies of social and public growth/development. In this sense it is fundamental to identify the different interests that may be involved in its practice such as politic, economic and educational aspects as well as the high power of convocation that it has in any of its various form of expressions - e.g. as a show, professional practice or free time and taking into considerations their dynamics and interactions. Following the ideas proposed by Dunning (Elías and Dunning, 1986), the image of the sport is idealized and mystified tearing it apart from the social ground that gives purpose to it. For these reasons the focus of this work is to study the sport, and in particular kid's football, as a social agent. The present work is embedded in the research project "Fútbol infantil y gobierno de la infancia. La Organización Nacional del Fútbol Infantil en el Uruguay (1968-2015)", whose aim is to generate a descriptive study of kid's football as governance of childhood, that starting off analysis of the notions of body and sport is able to identify, in kid's football, which sport for which infancy?.
\end{abstract}

Keywords: kid's football, childhood, governance, sport.

\title{
Resumo
}

No marco da relação que historicamente tem mantido a educação física com o esporte, se faz difícil não atender o significado do futebol infantil como prática cultural e fenómeno social em relação com a política pública na agenda de investigação atual. $O$ esporte apresenta-se como um fenómeno cultural, ao mesmo tempo que se apresenta fundamento recorrente ao momento de falar de desenvolvimento social e de políticas públicas. Neste sentido torna-se relevante visualizar os diversos interesses que o atravessam, desde as facetas política, económica, educativa e o grande nível de convocação e impacto que supõe a prática desportiva em suas diferentes formas de expressão -como espectáculo, como prática profissional ou de esporte no tempo livre numa primeira divisão dinâmica e interactuante-. Seguindo as linhas propostas por Dunning (Elías e Dunning, 1986), a 
imagem do esporte é idealizada e mistificada separando do terreno social que lhe outorga sentido, pelo que neste caso se propõe realizar uma análise do esporte em general do futebol infantil em particular como agência social. O apresente trabalho se enmarca no projeto de investigação "Futebol infantil e governo da infância. A Organização Nacional do Futebol Infantil no Uruguai (1968-2015)", que propõe gerar um estudo descritivo em relação ao futebol infantil em tanto governo da infância, que parta da análise das noções de corpo e esporte para poder identificar que esporte para que infância no futebol infantil.

Palavras-chave: futbol infantil, esporte, governo, infancia.

\section{Introducción}

Estudiar las posibles relaciones entre deporte y sociedad en el fútbol infantil supone: en primer lugar reconocer el recorrido histórico del deporte en la cultura de las sociedades modernas y el fenómeno masivo del fútbol infantil en el Uruguay superando las limitaciones que implica “conceptualizar los «objetos» del pensamiento sociológico como estáticos por un lado, y como aislados e independientes de las relaciones en que participan, por el otro." (Elías y Dunning 1986: 21) En segundo lugar, entender al deporte en general, y el fútbol infantil en particular (para este trabajo), como una de las agencias de socialización más importantes en la actualidad. El fútbol infantil en el Uruguay, aparece como práctica no obligatoria que nuclea a más de 45.000 niños y niñas vinculados a la Organización Nacional del Fútbol Infantil (en adelante ONFI), según cifras presentadas en el año 2017 por la secretaría de dicha organización. Se estima que el trabajo en relación a los actores que sostienen el movimiento (familiares, orientadores técnicos, dirigentes, etc.) hace que la cifra se eleve significativamente. Por lo tanto, es objeto del trabajo relacionar el fenómeno del fútbol infantil con los conceptos política, infancia y deporte a la vez que colaborar en revisar, cuestionar y construir el marco de referencia para hablar de la infancia y deporte desde el discurso de la organización en tanto parte de la política pública nacional (desde la mirada a los documentos fundantes de la creación y reglamentos y programas que configuran el contexto de su sentido). Análisis que se visualiza como necesario si tenemos en cuenta el peso que puede implicar el fútbol infantil en la construcción de la infancia y las representaciones de ésta. Para ello se propone pensar la política pública, entendida como 
"símbolos, estatutos de legitimidad, tecnologías políticas, formas de gubernamentalidad e instrumentos de poder que a menudo ocultan sus mecanismos de funcionamiento" (Shore, 2010: 21) y que vinculan para el caso las nociones de infancia, cuerpo, deporte y la relación con los derechos humanos como bases que por momentos fundamentan el fenómeno.

Es preciso considerar que el orden social y político no es posible entenderlo natural o neutral (esto es parte esencial en las representaciones sociales para develar una de las estrategias o funciones ideológicas esenciales del deporte de la que el fútbol infantil no escapa).

Esto exige aceptar que ha sido construido sobre la base de relaciones sociales, culturales y de poder. Supone además ser conscientes que otro orden ha sido excluido y que un determinado orden del discurso ha sido establecido. Es decir, el orden social y político que parece "natural" es, en realidad, el resultado de prácticas específicas, momentáneas y contingentes, en las que los discursos desempeñan una función legitimadora clave. En efecto, en el campo político se juega lo justo e injusto, la administración del sentido común colectivo, la gestión de los asuntos públicos y las prácticas de gobierno (Guerrero y Vega, 2015)

\section{En relación al deporte como tema de los estudios sociales y culturales}

El deporte como tema de interés de los estudios de las ciencias sociales y humanas surge en un contexto moderno que parte de la posibilidad de entenderlo en tanto práctica cultural y humana, respondiendo a la tensión existente en ese campo en el que a su vez se desarrolla. De todas las variables con las que se presenta el deporte en tanto eje temático de los estudios sociales y culturales, claramente se destaca la posibilidad de comprenderlo parte de las sociedades modernas. Las relaciones que giran en torno al deporte, los actores que son identificados por los estudios que lo tematizan, las disputas e intereses que afectan cada práctica, lo configuran como una institución cero en tanto es cargado de sentido y corresponde con las hegemonías discursivas del contexto en el que se lo analiza (Guedes, 1977). En tanto parte constitutiva de la sociedad y como espacio productor de alteridades, Archeti (1985) abre la posibilidad de reflexionar sobre la sociedad y el deporte como 
espacios dinámicos en el que suceden diversas relaciones que son factibles de ser estudiadas por las ciencias sociales y humanas (más allá de las ciencias biológicas que mantienen un predominio en los estudios relacionados al campo del deporte). Los grandes espectáculos y eventos deportivos, la creciente y constante relación del deporte con el mercado que suponen y fomentan en un contexto global, así como también el lugar que ocupa el deporte en las fundamentaciones de las políticas públicas hacen que el mismo tome un lugar central en la agenda pública y en la agenda de la investigación actual. En este sentido el deporte se presenta desde una mirada marxista en la intersección de la institución cotidiana del cuerpo, el aparato escolar, el espectáculo deportivo y los medios de comunicación de masas ocupando un papel importante en la lucha de clases (Brohm, 1993). Este marco de perspectivas, que en el devenir histórico han sido ampliadas y profundizadas, habilita pensar el fútbol infantil en el Uruguay como espacio donde se establecen relaciones particulares entre políticas, cuerpo, infancia y deporte. Sumando de esta manera al juego de la intersección, planteado en Brohm, la posibilidad de pensar el fútbol infantil como espacio de construcción y configuración de la infancia.

Partiré de explicitar que en el Uruguay el fútbol infantil aparece regulado por el estado en términos de "ente rector". Esto permite colocarlo fuera del funcionamiento federativo del deporte y las condiciones que ello supone. El trabajo podría aportar desde una perspectiva Nacional al estudio del campo del deporte en la región, reconociendo los procesos y las formas en que los deportes se referencian en tanto manifestación cultural y “como o esporte históricamente foi se sociabilizando e tornando-se referencia nas práticas cotidianas dos sujeitos, sobretudo nos espaços urbanos" (Ribeiro y de Almeida Ribeiro, 2015: 110).

El deporte en la región aparece como un fenómeno reiterado al hablar de desarrollo y de políticas públicas por la relevancia que toman los diversos intereses que lo atraviesan desde las facetas política, económica, educativa y el gran nivel de convocatoria que supone la práctica deportiva en sus diferentes formas (como espectáculo, como práctica profesional o del deporte en el tiempo libre). Si se propone abordarlo desde una perspectiva crítica y de reflexión constante, no se puede desanudar de la colonización futbolística del espacio social 
que representa para este deporte la Federation Internationale de Football Association (FIFA) y cómo el fútbol tiende a suplantar y cooptar prácticas populares y juegos de cada cultura. Para que ello suceda el fútbol conquista el espacio social y se apropia simbólicamente del mismo a través de los diversos marcos institucionales que lo representan en el espacio físico o territorio en el que "el fútbol tiene lugar" (Carrión, 2017: $338)$.

\subsection{Una mirada al contexto Uruguayo}

El fútbol infantil en el Uruguay presenta una particularidad que difiere de los diferentes países de la región y es que desde la creación de la Comisión Nacional de Baby Fútbol en el 1968 mantiene una dependencia con el estado en tanto política social en sus inicios siendo regulada por la Comisión Nacional de Educación Física. Los movimientos sociales y culturales, así como los discursos y configuraciones de la infancia hacen que en el 2001 dicha comisión pase a nombrarse Organización Nacional de Fútbol Infantil (ONFI) acompasándose con un lenguaje que refiriera mejor a la franja etaria que convoca (entre 6 y 13 años) y se mantenía el vínculo estatal mediante el encuadre de dependencia del Ministerio de Deporte y Juventud (actualmente inexistente.) Actualmente la ONFI depende de la Secretaría Nacional del Deporte (directamente vinculada a Presidencia de la República desde el año 2016) por lo que se podría decir que la política pública en relación al deporte toma cierta centralidad. ${ }^{1}$ A su vez en este marco institucional, a partir de la Ley $\mathrm{N}^{\circ} 19.331^{2} /$ artículo 12, quién responde ante la solicitud de información o abordaje del deporte ante la solicitud del Poder Legislativo es el Ministerio de Educación y Cultura. Pese a ello no cuenta con un presupuesto estable y relacionado a la magnitud de la misma. Este dato aparece como fundamental al momento de reconocer que "las políticas sociales no pueden ser el resultado de entusiastas planteamientos voluntaristas. Por el contrario, son la expresión fundamental de los propósitos de la sociedad y de las posibilidades económicas para alcanzarlos, que obviamente son realidades inseparables." (Carambula,

\footnotetext{
1 Por más referencias de la Organización Nacional del Fútbol Infantil y de su historia visite: http://www.onfi.org.uy

2 Ley de Creación de la Secretaría Nacional del Deporte. Disponible en: https://legislativo.parlamento.gub.uy/temporales/leytemp2346915.htm
} 
2009: 3) A los recursos destinados por parte del estado para sostener al fútbol infantil, se le suma la necesidad imperiosa de discutir a nivel académico estas relaciones entre política pública, deporte, derechos humanos y otros fundamentos que se utilizan para sostener la presencia de las políticas vinculadas al deporte a nivel Nacional y que muchas veces sustituyen los análisis de sentidos y significados de los contenidos y conceptos centrales de la política pública reduciendo así el eje de discusión.

En relación a los elementos manejados hasta el momento, los datos que proporciona el ente rector, colocan al fútbol infantil en el Uruguay como una práctica de gran impacto y alcance. Más aún, si se tiene en cuenta que es una práctica no obligatoria. De un total de 169.430 niños y niñas entre 6 a 13 años el fútbol infantil mantiene una cantidad de niños fichados de 48.144 que corresponde al 28, 42 por ciento del total. ${ }^{3}$ Siguiendo esta línea de pensamiento, las relaciones que suceden en dicho espacio impactan significativamente sobre la configuración y construcción de representaciones de la infancia. El recorrido de los documentos oficiales a lo largo de la historia, nos encasilla en la mirada del baby fútbol ${ }^{4}$ como espacio de aprendizaje de lo que sucederá a futuro en la ocasional profesionalización deportiva del niño y transmisión de las lógicas en las que funciona ésta. Esto se articula directamente con la información que cotidianamente estamos acostumbrados a recibir del mundo del deporte. Este supuesto aparece en el reglamento del ente rector, desde su creación en 1968, bajo diferentes modalidades que se sostienen en la actualidad bajo las diferentes disposiciones heredadas. En el año 1980 la Comisión Nacional de Educación Física realiza una publicación denominada "Reglamento para la práctica de Baby - Fútbol, Tomo I.” En ésta, se detalla el marco regulatorio para los campeonatos y las funciones del ente rector; se destaca para la relación establecida en el presente trabajo, el período de pases entre los niños de los clubes. En los art. 13, 14 y 15 del reglamento general para las ligas, se manifiesta que existirá un plazo para presentar las listas de retención de jugadores, estableciendo que los jugadores no retenidos serán considerados como libres. "Cada año las Ligas de baby Fútbol de todo el país habilitarán un período de Pases al que podrán acceder

\footnotetext{
${ }^{3}$ Datos brindados por la Secretaría de ONFI para la presentación de la Organización en el marco del trabajo conjunto UdelaR - ONFI 2017.

${ }^{4}$ Actualmente denominado fútbol infantil desde el cambio de denominación del ente rector en el 2001.
} 
los jugadores con carácter de libres o aquellos a los que su Club les concede el pase a otra Institución. (Comisión Nacional de Educación Física, 1980: 7) En este tipo de ejemplos radica la asociación directa del fútbol infantil con la noción de semillero o cantera búsqueda de los héroes deportivos; siendo esta otra posibilidad de abordar, analizar y evidenciar la relación entre fútbol infantil y gobierno de la infancia.

\subsection{Hacia la visibilidad del fútbol infantil como gobierno de la infancia}

El fútbol como fenómeno en el marco de la globalización deportiva constituye uno de los espectáculos con más reconocimiento a nivel mundial, siendo igual de impactante las diferentes formas de consumo. El espacio deportivo y en particular el fútbol infantil en el Uruguay, aparece bajo la órbita del ente rector estatal, por tanto, puede ser considerado como práctica masiva con el ingreso de variados actores en el campo en disputa y espacio de formación que puede tornarse privilegiado. Dicho planteo requiere la revisión de dos cuestiones esenciales: A) la asociación constante entre deporte y educación, deporte y salud o deporte e inclusión y su relación con los derechos humanos y en particular a los derechos de la infancia. B) La relación con la creciente globalización del deporte como espectáculo que según basa su crecimiento en la relación de tres aspectos: el deporte como fuentes de emoción agradable; como medio de identificación colectiva; y como elemento clave que dota de sentido la vida de muchas personas. (Elías y Dunning, 1986)

Estos elementos transversalizan la infancia, en tanto las representaciones del cuerpo que se constituyen en las relaciones sociales, y en los procesos educativos, determinan la posición-lugar de éste en la sociedad, generándose así un saber cultural aplicado al cuerpo, que para el caso, se torna infantil, deportivo y masculinizado (no abordaremos este último punto en el actual trabajo si bien es reconocido como elemento central por la investigación que lo enmarca). Estos puntos se nuclean en los documentos del ente rector y encuentran en el sinónimo “jugador” la articulación en una clara asociación con la práctica profesional del fútbol entre cuerpo, infancia y cultura deportiva. A modo de ejemplo se extraen del reglamento general para las ligas

Art. 7 La vestimenta que utilizarán los jugadores será la que se utiliza comúnmente para la práctica del fútbol, o sea que las camisas, camisetas, 
pantalones cortos y medias deberían ser iguales en todos los componentes del equipo, con excepción del golero que utilizará buzo cuyo color deberá ser notoriamente distinto al color de la camisa del resto del equipo.

Art. 8 Las camisetas, así como el buzo del golero, habrán de lucir, en la parte posterior, un número de identificación del jugador. Dicho número deberá ser anotado en el formulario del partido junto al nombre de su portador. El golero podrá utilizar como parte de su vestimenta pantalón buzo. (Comisión Nacional de Educación Física, 1980: 6)

Este apartado además de colocar algunos elementos identificatorios de la práctica de fútbol en el cuerpo del niño (reglamentado ahora), presenta un ejemplo de los reiterados casos donde el reglamento sustituye constantemente la palabra niño por jugador en una suerte de paralelismo o imitación entre infancia (jugador) - adultez (jugador de fútbol). El reglamento en este punto se propone como líneas posibles en la idea de dispositivo, en tanto trama que se configura desde relaciones de fuerzas formando una red de poder, saber y subjetividad. Reconociendo entonces que "Un dispositivo produce subjetividad, pero no cualquier subjetividad.” (Fanlo, 2011: 4)

En la noción de educación y en su carácter de transmisión cultural Fattore y Caldo, (2011) se encuentra una ardua tarea de revisión y promoción de nuevos enfoques que permitan visualizar una tensión en la educación como

[...] figura de la continuidad y del futuro, y en este sentido es conservación de la tradición, es anticipación, proyecto, prescripción, planificación, fabricación que pareciera depender de nuestro saber, de nuestro poder, de nuestra voluntad. Al mismo tiempo, y por suerte, es discontinuidad y porvenir; porvenir entendido como figura del acontecimiento, de la irrupción de lo no esperado, de lo no previsto, de aquello que no se deja atrapar por las categorías de las que disponemos ni por las prácticas que desplegamos. (Fattore y Caldo, 2011: 11).

Desde este enfoque se puede entonces visibilizar elementos de impacto en la configuración de la infancia y el papel que juega (como agencia social) el fútbol infantil en 
los espacios de transmisión cultural y conformación de identidades. Haciendo referencia a Michel Foucault, Bustelo destaca la noción de biopolítica como forma de manifestación del poder, "puesto que reglamenta la vida social desde adentro de cada persona" (Bustelo, 2007: 60) suponiéndolo de esta manera más efectivo conformándolo como parte vital del comportamiento a través de las normas y valores que los individuos internalizan. Es así que la relación deporte - infancia toma relevancia en el marco concreto de análisis en el que se visualizan los flujos de información y los medios masivos de comunicación así como el desarrollo de la tecnología, y el uso del tiempo libre, impactando de forma sustancial en la construcción de la infancia. En este sentido la infancia supone pensar a niños y adolescentes como "consumidores y dinamizadores del consumo" (Bustelo, 2007: 59). El fútbol infantil no escapa a esta realidad que se potencia desde la capacidad de convocatoria que mantiene, ya sea en el rol de practicante o en el rol de espectadores de eventos deportivos haciendo del mismo

un campo de disputa simbólica donde concurren diversos actores que buscan, incluso de manera inconsciente, definir los sentidos "verdaderos" sobre los que se asienta su identidad como individuos y como grupo social. En esa medida, el fútbol sería también escenario de conflicto entre grupos sociales que buscan imponer sus sentidos a los otros con los cuales se hallan en competencia y, por lo tanto, un escenario donde se disputa la hegemonía. (Alabarces, 2003:28)

Los conceptos de deporte, infancia y cuerpo son articulados por los discursos que conforman la idea de gobierno de la infancia en relación con el fútbol infantil. Foucault dedica varias de sus obras al análisis del gobierno en las sociedades modernas. En este sentido el concepto de gobierno se va transformando y complejizando al final de su obra y en este entramado entiende al gobierno como técnica general que pasa desde el control de si, hasta el control de las poblaciones. Esta noción ya tenía en la obra de Foucault "Historia de la sexualidad III", una relación con la noción de "cultivo de si” caracterizándolo como el cuidado de sí mismo a partir de nociones del individualismo; suponiendo que es desde uno mismo, desde una inquietud que funda su necesidad, donde gobierna su desarrollo y se organiza su práctica (Foucault, 1984). El cuerpo toma relevancia como espacio de 
inscripción de lo político y de gobierno de si y de los otros. Siendo la noción médica respecto al cuerpo la que históricamente ha definido los discursos y los parámetros desde donde se configura; el deporte por tanto emerge como la institucionalización del discurso que puede otorgar bajo el velo de otros fundamentos una noción de eficiencia y eficacia corporal, que por momentos parece encubierta.

La infancia es colocada entonces en el fútbol infantil, en el marco de una cultura deportiva, un cuerpo deportivo, una moral deportiva desde donde se organiza la misma práctica (Rodríguez, 2002). La posición que se toma desde la política pública no es neutra y no debería naturalizar las relaciones que en el espacio del fútbol infantil se generan. Esto lo torna un espacio de constantes luchas con la finalidad de trascender la mirada de la práctica deportiva como derecho humano y única fundamentación sin profundizar en lo que ello implica. Esta intención de neutralidad en torno al deporte o a quienes lo regulan ya estaba marcada en el art. 6 de los "Estatutos de la Comisión Nacional de Baby Fútbol” recogidos en una publicación de la Comisión Nacional de la Educación Física del año 1976, donde se establece para la misma, una finalidad esencialmente deportiva social y cultural que "se mantendrá rigurosamente alejada de toda actividad política y/o filosófica" (Comisión Nacional de Educación Física, 1976: 9). En este sentido no es nada casual la relación que mantiene el año de dicha publicación de la Comisión Nacional de Educación Física con el período entre los años 1973 y el 1976 del Uruguay, denominado por el historiador B, Nahum (1999) “Afirmación de la Dictadura”. En este período se aprueba la "Ley orgánica Militar (...) principal elaboración teórica del Gobierno destinada a justificar el Golpe y sus secuelas" y se auto denomina la dictadura como proceso cívico militar 5 . (Nahum, 1999:172)

3. De los documentos del ente rector, al habitus que supone el fútbol como estructura estructurada y estructurante

\subsection{Del cometido de la ONFI}

${ }^{5}$ Este proceso se da a partir del primer y segundo Acto Institucional que irían supliendo a la Constitución desde la suspensión de las elecciones y la conformación del Consejo de la Nación (con integración cívico militar) quien tenía la potestad de nombrar al Presidenete de la República, a los miembros del consejo del estado, "de la Suprema Corte de Justicia, Tribunal de lo Contencioso Administrativo y Corte Electoral" (Nahum, 1999: 173) 
En el artículo 1 del "Reglamento general de organización y funcionamiento" vigente se propone que:

"La Organización Nacional de Fútbol Infantil (ONFI) funciona en la órbita del Ministerio de Turismo y Deporte, ejerciendo, en forma exclusiva, la rectoría del fútbol infantil entre los 6 y 13 años de edad, en todo el territorio nacional. Tiene como finalidad fomentar una actividad beneficiosa para la formación física y moral de la niñez. Puede coordinar, acordar o convenir su actuación con toda clase de instituciones públicas o privadas a efectos de cumplir con los fines mencionados, procurando la continuidad de la práctica deportiva de los niños cuando se conviertan en adolescentes." (Ministerio de Turismo y Deporte - ONFI, 2015)

En primer lugar hay algunos elementos que interesa destacar en relación a lo que Llobet (2011) denomina "proceso de institucionalización" por los que atraviesan los derechos del niño, niña y adolescente a través de la Convención Internacional de Derechos del Niño en tanto "instrumento de derechos humanos y marco de especificación de la ciudadanía infantil.” (Llobet, 2011: 447) Este punto supone una serie de desafíos analizados por la autora entre los que podemos encontrar aquellos que guardan directa relación con la problemática puesta en escena y con la participación en el contexto de las prácticas corporales y en particular el fútbol infantil. Por un lado en dicho proceso de institucionalización de los derechos de los niños, niñas y adolescentes se puede visualizar un gran activismo político por parte de estados y particularmente adultos para alcanzarlos. (Llobet, 2011) Son los actores políticos, adultos, quienes en nombre de los niños definen las acciones en relación al fútbol infantil desde la noción que para ellos representan los niños y la infancia. Estos instrumentos que se proponen en un marco de universalidad como lo son los derechos humanos, ${ }^{6}$ si bien habilitan garantías universales que promueven la

\footnotetext{
${ }^{6}$ Fundamento esencial de la Secretaría Nacional del Deporte, de quien depende la ONFI, al momento de hablar de la Educación Física, las actividades físicas, el deporte y la recreación. Este discurso se puede observar en la presentación del Plan Nacional Integrado de Deporte de la ministra de turismo y deporte "El PNID constituye un camino hacia la recuperación de los éxitos que el país supo disfrutar, pero sobre todo un instrumento para garantizar el derecho de los ciudadanos de realizar actividad física." (Plan Nacional
} 
transformación social y el respeto de la humanidad toda, no pueden entenderse neutros. Cada carta, cada reunión, cada declaración que se transforman en acuerdos universales, a la vez que son aprobados por cada país miembro merecen una revisión que nos permita seguir reflexionando y no estancarnos en acciones fundamentadas externamente y aisladas de una visión en relación al contexto particular y social de producción. ${ }^{7}$

En esta misma línea como segundo aspecto a resaltar, contribuye la idea de Leopold (2014) en relación a la construcción de la infancia, donde a partir del reconocimiento a la tesis central de Ariès en la que destaca lo que denomina como -inexistente sentimiento de infancia- en la edad media, se identifica

(...) cierta procesualidad sociohistórica que en su devenir hará emerger y consolidará la categoría infancia en el mundo moderno como resultado de una lenta transformación de actitudes, sentimientos y modalidades de relacionamiento de los adultos con respecto a los niños. (Leopold, 2014:

Este enfoque propone una mirada donde hablar de participación y derechos humanos se enfrenta a los desafíos de las desigualdades en la infancia y de cómo se ponen en juego las políticas y acciones de gobierno frente a un problema social, ${ }^{8}$ en este caso vinculando infancia y deporte, en una suerte de deportivización de la infancia. Más específicamente, la Comisión Nacional de Baby Fútbol propone dictar "normas para reglamentar esta actividad en sus aspectos físico, moral y social." (Comisión Nacional de Educación Física, 1976: 6) Y dentro de sus fines se destacan la estimulación de la práctica del Baby fútbol, la organización de los campeonatos Nacionales de clubes campeones y selecciones departamentales, organización y participación en certámenes de índole internacional y oficializar y controlar los torneos que se organizan en todo el territorio nacional por las departamentales y ligas afiliadas. (Comisión Nacional de Educación Física,

Integrado de Deporte, 2012: 3) Este documento se constituyó como la base del trabajo de la Secretaría Nacional del Deporte a partir de su creación.

${ }^{7}$ Lo que permitiría al decir de Fonseca y Cardarello (2005) comprender a través de qué procesos se visibilizan socialmente en un contexto histórico y político que los propone en tanto realidades en las que el estado debería intervenir (Villalta, 2016) 
1976) En el artículo 1, inciso A del Reglamento de funcionamiento actual se intenta superar esta mirada reconociendo al momento de "Organizar, dirigir, supervisar y estimular la práctica del fútbol infantil en todo el territorio nacional, poniendo énfasis en los aspectos sociales, educativos y recreativos sobre los competitivos" (Ministerio de 2015)

En segundo lugar y profundizando la temática en torno a los derechos humanos desde el planteo de Fonseca y Cardarello (1999) se desprende que la reflexión sobre los mismos descontextualizada y de forma abstracta hacen que poco signifiquen. Por lo que emerge la importancia sobre cómo estas nociones que resultan de los derechos humanos, impactan y configuran consecuencias particulares según las relaciones de poder que se establecen en contextos históricos específicos y son expresadas a su vez en categorías precisas dependiendo su resultado de las relaciones de poder que las forjan. Aquí es preciso retomar del artículo 1 citado anteriormente, que la ONFI "Puede coordinar, acordar o convenir su actuación con toda clase de instituciones públicas o privadas a efectos de cumplir con los fines mencionados, procurando la continuidad de la práctica deportiva de los niños cuando se conviertan en adolescentes." (Ministerio de Turismo y Deporte - ONFI, 2015) En esta línea se visualiza una segunda tensión que se expresa por ejemplo en el vínculo del estado con los sponsors privados para lograr ser visibilizados o sostener económicamente la organización y desarrollo de los campeonatos. Mirado desde los paradigmas en relación a las políticas sociales en América Latina que aborda Franco Rolando (1996), se vincula directamente a la co financiación que resalta en el paradigma emergente de las políticas sociales, donde el Estado ya no es el único actor de la política social y la cofinanciación puede surgir de empresas bajo diversas modalidades. (CEPAL, 1996) Esta suerte de frente discursivo que resulta de la negociación de los interesados en producir en torno a una misma temática, se transforma en una posibilidad de movilización política sobre acuerdos y bases amplias y eficaces o por el contrario puede alimentar imágenes que tienden a naturalizar discursos que poco tienen que ver con el objeto de la política. En este sentido se puede plantear

que se os ativistas dos direitos humanos não mantêm um certo distanciamento em relação a este jogo discursivo, correm o risco de montar 
programas que não apenas deixam de alcançar os seus objetivos mas, pior que isto, produzem novas formas de exclusão (Fonseca y Cardarello, 1999: 85)

Bajo esta mirada los discursos de las políticas en relación al fútbol infantil y su forma de regulación, suponen ser comprendidos como escenarios centrales de producción de infancia, es decir, aparecen construyendo imágenes y discursos sobre dichos grupos sociales capaces de influir por tanto en sus condiciones de existencia bajo diferentes mecanismos de los cuales se destacan los universales simbólicos. (Llobet, 2012)

En tercer lugar y vinculado a la idea de diversos modos de gobierno, no es menor que aparezca la finalidad de la Organización en "fomentar una actividad beneficiosa para la formación física y moral de la niñez." Y procurar la "continuidad de la práctica deportiva de los niños cuando se conviertan en adolescentes." (Ministerio de Turismo y Deporte ONFI, 2015) Ante la pregunta entonces respecto a qué práctica deportiva es de la que estamos hablando, los cometidos de la ONFI en el artículo 2 de su reglamento de funcionamiento nos permiten la respuesta; que se relaciona directamente con la organización del fútbol adulto y profesional. ${ }^{9}$

En este sentido se cruzan dos ejes sobre los cuales se imbrica directamente la política en torno al fútbol infantil. El primero tiene que ver con la (siempre) intención de incidir en el cuerpo de la infancia. Y el segundo eje aparece en la idea de perpetuación de la práctica deportiva como buenas prácticas sin cuestionar que significa ello. Esta noción se refuerza si la analizamos en el marco de la perspectiva de Bourdieu, donde el habitus es asegurador de las experiencias pasadas que "registradas en cada organismo bajo la forma de esquemas de percepción, de pensamientos y de acción, tienden, con más seguridad que todas las reglas formales y todas las normas explícitas, a garantizar la conformidad de las prácticas y su constancia a través del tiempo." (Bourdieu, 2007; 88-89) El aparente sentido de neutralidad de las prácticas, omite muchas veces bajo el velo de los derechos humanos

\footnotetext{
${ }^{9}$ Dentro de los que podemos destacar "Organizar o autorizar la realización de juegos nacionales, regionales, locales o de cualquier otra naturaleza dentro del ámbito de actuación." Y "Acordar, esponsorizar o comercializar con cualquier entidad, pública o privada, todas aquellas actividades que se crea convenientes para su mejor desarrollo.” (Ministerio de Turismo y Deporte - ONFI, 2015 Art. 2)
} 
un análisis crítico de las acciones propuestas por la propia política y sus alternativas en tanto prácticas de gobernabilidad, reduciendo así el sentido educativo de las mismas y su relación con el conocimiento.

\section{A modo de apuntes para el diálogo}

Los espacios de deporte infantil, muchas veces son sostenidos y motivados por la trayectoria de grandes iconos deportivos considerados héroes del deporte, distanciándose de las posibildiades variadas y diversas que suponen comprenderlo en tanto práctica cultural. O mejor dicho, en tanto práctica culutral, al fútbol infantil le cuesta dar a conocer prácticas que respondan menos a la relación directa con el deporte profesional. Por lo que a partir de identificar los elementos, signifcados y formas de impactar de las políticas que se inscriben en el cuerpo, se podría realizar el ejercicio de pensar alternativas en los modos de gobierno. Dentro de las finalidades de la Organización priman aquellas ideas que organizan las competencias deportivas y se vinculan claramente con la forma en que el deporte profesional se organiza. Cabe preguntarnos si pensando en el fútbol infantil en tanto práctica cultural y espacio de formación no existen otros elementos que puedan ser tenidos en cuenta desde la política social que lo regula. Claramente es la propia organización en diálogo con los diferentes actores que la conforman y con quienes articula, la que debe realizar dicho ejercicio, impactando en las formas y contenidos que se propone desarrollar. Lejos de ser una revisión histórica profunda, esta propuesta puede dar lugar al diálogo que permita comprender desde dónde se fundamentan las prácticas que se desarrollan. Sin lugar a dudas que la lógica de mercado que regula y a la vez presupone el fenómeno del fútbol en tanto práctica gloablizada, encorceta el funcionamiento de la Organización Nacional del Fútbol Infantil y las acciones que desarrolla. Pero en tanto regulación estatal debemos permitirnos la posibillidad de pensar más allá de la lógica donde los campeonatos, los dirigentes, los jugadores y ya no los niños pasan a configurar la centralidad de la propuesta. Las inversiones privadas sin lugar a dudas terminan siendo un sostén fundamental para la organización, pero los interéses que se mantienen desde los inversores lejos están de ser los que se proponen vinculados a la recreación y a la educación como se propone en el reglamento de funcionamiento de la misma. Articulando en este punto la 
mirada del deporte en tanto agencia social, es esencial avanzar en un diálogo político y pedagógico del deporte, potencialmente emancipador. Para ello no puede estar ausente la reflexión desde marcos que articulen teoría, entorno, participantes, de lo contrario se estaría recayendo en una praxis neoliberal y mercantilista reproductora de una relación de fuerzas que no colabora en conformar un estado de bienestar que supere las desigualdades del sistema a la hora de pensar la relación deporte y políticas públicas.

Mirar el fútbol infantil desde la mítica deportiva globalizada, podría llevarnos a perpetuar y reproducir formas y modos de ver a la infancia que a partir del seguimiento de modelos y héroes deportivos, comerciales y vendibles a nivel mundial que luchan por la igualdad de los niños en el mundo; por el contrario acentúen las desigualdades económicas, sociales y profundas brechas culturales existentes en América Latina.

En la noción de campo como espacio de luchas que propone Bourdieu a lo largo de su obra, las estructuras que configuran el fútbol infantil podrían verse afectadas por otras formas de transitar las prácticas deportivas en la infancia, reconociendo en la misma la potencialidad genérica ante la presencia del habitus estructurante del fútbol espectáculo. Pero para ello debe existir un verdadero interés por develar la aparentemente neutral, que permita pensar en transformar las estructuras propuestas desde el campo de juego, reglamentos, formas de desarrollo de encuentros, etc. pensando en una práctica corporal que reconozca a los actores -y a los actores infantiles- como productores de prácticas. Favoreciendo así, espacios de diálogo donde la transmisión cultural se pone en juego junto a la realidad social que genera la participación infantil como motor fundamental y "centro de interés" de la propuesta.

La regulación y control de los cuerpos infantiles fue objetivo central del discurso pedagógico moderno. Para esto la escuela delimitó a lo largo de la historia el cuerpo infantil (Scharagrodsky, 2013), ahora bien; cuando analizamos las políticas públicas fuera de la institución escolar ¿existen diferencias que propongan alternativas o son nuevas formas de delimitación de un cuerpo infantil en este caso en una escuela deportiva? 


\section{Referencias bibliográficas}

Alabarces, P. (2000). Peligro de gol. Estudios sobre deporte y sociedad en América Latina, CLACSO, Buenos Aires.

Alabarces, P. (2003). Futbologías. Fútbol, identidad y violencia en America Latina. CLACSO, Buenos Aires.

Arancibia, J. P. (2004). El lenguaje y lo político. En: Revista Comunicación y medios, Chile, n. 15, p. 67-83.

Archetti, E. (1985). Fútbol y ethos en Monografías e Informes de Investigación. Serie Investigaciones, $\mathrm{N}^{\mathrm{o}}$ 7, FLACSO, Buenos Aires.

Bayce, R. (2013). El Deporte como Agente Educativo. En: Foro CIPES 2013: El Poder de Educar. Otros protagonistas: Medios de comunicación - Deporte. Montevideo: CIPES. Publicado el 16 de julio. Disponible en: (https://www.youtube.com/watch?v=E4i0f80Q_Cg).

Bourdieu, P. (1984). Sociología y cultura. México D.F.: Editorial Grijalbo, 1990.

Bourdieu, P. (2007). El sentido práctico. Buenos Aires: Siglo veintiuno editores, 1980.

Brohm, J.. (1993). 20 Tesis del deporte. En: Brohm, J. M. y Barbero, J. I. Materiales de Sociología del deporte (Genealogía del Poder). Madrid: Ediciones de la Piqueta, p.39-57.

Bustelo, E. (2007). El recreo de la infancia. Argumentos para otro comienzo. Buenos Aires: Siglo XXI Editores.

Carambula, M. (2009). Política y sociedad más y mejores políticas sociales en el Uruguay. Las Piedras: [s.n.] Disponible en: (http://www.espectador.com/documentos/carambulasocial.pdf) Acceso: 15/12/2016.

Carrión, F. (2017). El Fútbol coloniza el espacio social. Soto, R. y Fernández, O.. ¿Quién raya la cancha?. Buenos Aires: CLACSO, p. 337-354. 
Comisión Económica para América Latina y el Caribe [CEPAL] (1996) Los paradigmas de la política social en América Latina. [s.1]: No sometido a revisión editorial.

Comisión Nacional de Educación Física (1976) Comisión Nal. de Educación Físcia Comisión Nal. de Baby Fútbol. TOMO II, Montevideo, CNEF.

Comisión Nal. de Educación Física (1980) Reglamento para la práctica de baby fútbol. TOMO I, Montevideo, CNEF.

Elías, N. y Dunning, E. (1986). Deporte y ocio en el proceso de la Civilización. Buenos Aires: Fondo de Cultura económica, 1992.

Fattore, N. y Caldo, P. (2011). Transmisión: una palabra clave para repensar el vínculo pedagogía, política y sociedad. En: VIII Encuentro de Cátedras de Pedagogía de Universidades Nacionales Argentinas. La Plata: Memoria Académica [en línea] Universidad Nacional de la Plata- Facultad de Humanidades y Ciencias de la Educación. Disponible en: Memoria Académica (http://www.memoria.fahce.unlp.edu.ar/trab_eventos/ev.962/ev.962.pdf).

Fonseca, C. y Cardarello, A. (1999). Direitos dos mais e menos humanos. En: Horizontes Antropológicos. Porto Alegre, v. 5, n.10, p. 83-121. Disponible en: (https://dx.doi.org/10.1590/S0104-71831999000100005).

Foucault, M. (1970). El orden del discurso. Buenos Aires: Tusquets Editores, 1992.

Foucault, M. (1984). Historia de la sexualidad III. México: Siglo XXI Editores, 1987.

Frigerio, G., Diker, G., Mendoza, S. (2009). Los proyectos de inclusión educativa y la problemática de su evaluación. Buenos Aires: Programa EURO social-Educación, Proyecto "Indicadores y procedimientos para el monitoreo y evaluación de proyectos de inclusión y promoción educativa".

Guedes, S. (1977) O Futebol Brasileiro: Institucao zero. Master's diss. URFJ, Rio de Janeiro.

Guerrero, N. y Vega, M. J. (2015). Reflexiones teóricas sobre discurso político e ideología : un análisis preliminar de las políticas de emprendimiento del gobierno de Piñera 
(Chile 2010-2014). En: Revista Uruguaya de Ciencia Política [en linea], v.24, n.2, p.105-120.

Le Breton, D. (1990). Antropología del cuerpo y modernidad. Buenos Aires: Nueva Visión, 2002.

Leopold, S. (2013) Los laberintos de la infancia Discursos, representaciones y crítica. Montevideo: Ediciones Universitarias, Unidad de Comunicación de la Universidad de la República, 2014.

Llobet, V. (2011) Las políticas para la infancia y el enfoque de derechos en América Latina: algunas reflexiones sobre su abordaje teórico. En: Fractal: Revista de Psicologia. Fluminense, v. 23, n. 3, p. 447-460, Set./Dez, 2011.

Llobet, V. (2012) Políticas sociales y ciudadanía. Diálogos entre la teoría feminista y el campo de estudios de infancia. En: FRONTERA NORTE. San Antonio del Mar, v. 24, n. 48, p. 7-36, julio-diciembre.

Ministerio de Turismo y Deporte - Organización Nacional de Fútbol Infantil (2015) Reglamento general de organización y funcionamiento. Uruguay: ONFI, 2015. Disponible en: (http://www.onfi.org.uy/onfi_mixto/index.php/reglamentos/883reglamento-de-organizacion-y-funcionamiento-2015). Acceso: 15/12/2016.

Ministerio de Turismo y Deporte (2012) Pla Nacional Integrado de Deporte. Montevideo: Ministerio de Turismo y Deporte - Dirección Nacional de Deporte.

NACIONES UNIDAS - CEPAL (2015). Documento informativo. Panorama Social de América Latina. CEPAL, 2015 Disponible en (http://www.clacsovirtual.org/mod/resource/view.php?id=6284)

Nahum, B. (1999). Breve historia del Uruguay independiente. Montevideo: Ediciones de la Banda Oriental.

Oszlack, O. y O’Donnell, G. (2007). Estado y políticas estatales en América Latina: hacia una estrategia de investigación. En: Acuña, Carlos. Lecturas sobre el Estado y las 
políticas públicas: Retomando el debate de ayer para fortalecer el actual. Buenos Aires: Proyecto de modernización del estado, jefatura de la nación.

Ribeiro, L. y De Almeida, G. (2015). Futebol como objeto de cultura. En: Cornelsen, E.; Augustin, G.; Da Silva, S. Futebol, linguagem, artes, cultura e lazer. Rio de Janeiro: Jaguatrica, p. 109-122.

Rodríguez, R. (2002). Deporte y quehacer pedagógico: una relación a pensar críticamente. En: http://www.efdeportes.com/ Revista Digital. Buenos Aires, Año 8, n. 55, Diciembre de 2002. (Ponencia presentada en el IV Encuentro Deporte y Ciencias Sociales, Buenos Aires, noviembre de 2002).

Scharagrodsky, P. (2013). Coordinación Autoral: Southwell, Myriam. El cuerpo en la escuela. En: Educ.ar (Explora Pedagogía). Porto Alegre. Disponible en (http://www.educ.ar/sitios/educar/recursos/ver?id=111067).

Señorans, J. (2015). La cara oculta del baby fútbol. Montevideo: Editorial Fin de siglo.

Shore, C. (2010). La antropología y el estudio de la política pública: reflexiones sobre la “formulación” de las políticas. En: Antípoda. Colombia, n. 10, enero-junio, p. 2149.

Villalta, C. (2008) La administración estatal de la minoridad y la infancia y las desigualdades. En: ESTUDIOS EN ANTROPOLOGÍA SOCIAL - CAS / IDES. Buenos Aires, v. 1, n. 2, p. 81-99.

Villalta, C. (2016). "Dispositivos jurídico-burocráticos para la gestión de la desigualdad de la infancia. Entre la incorporación controlada y la protección de derechos”. Clase 5 del Seminario Desigualdades y generaciones. Buenos Aires: CLACSO.

Villena, S. (2003) El fútbol y las identidades Prólogo a los estudios latinoamericanos. En: Albaceres, P. Futbologías. Fútbol, identidad y violencia en América Latina. Buenos Aires: CLACSO, p. 21-35. 